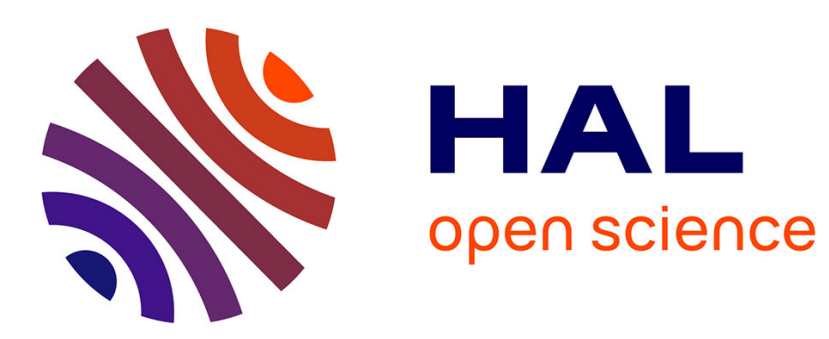

\title{
A conformal mapping algorithm for the Bernoulli free boundary value problem
}

\author{
Houssem Haddar, Rainer Kress
}

\section{To cite this version:}

Houssem Haddar, Rainer Kress. A conformal mapping algorithm for the Bernoulli free boundary value problem. Mathematical Methods in the Applied Sciences, 2015, 10.1002/mma.3708 . hal-01214261

\author{
HAL Id: hal-01214261 \\ https://hal.inria.fr/hal-01214261
}

Submitted on 13 Oct 2015

HAL is a multi-disciplinary open access archive for the deposit and dissemination of scientific research documents, whether they are published or not. The documents may come from teaching and research institutions in France or abroad, or from public or private research centers.
L'archive ouverte pluridisciplinaire HAL, est destinée au dépôt et à la diffusion de documents scientifiques de niveau recherche, publiés ou non, émanant des établissements d'enseignement et de recherche français ou étrangers, des laboratoires publics ou privés. 


\title{
A conformal mapping algorithm for the Bernoulli free boundary value problem
}

\author{
Houssem Haddar \\ INRIA, Ecole Polytechnique, Route de Saclay, \\ 91128 Palaiseau Cedex, France (haddar@cmap.polytechnique.fr) \\ Rainer Kress \\ University of Göttingen, Lotzestrasse 16-18 \\ D-37083 Göttingen, Germany (kress@math.uni-goettingen.de
}

October 13, 2015

\begin{abstract}
We propose a new numerical method for the solution of Bernoulli's free boundary value problem for harmonic functions in a doubly connected domain $D$ in $\mathbb{R}^{2}$ where an unknown free boundary $\Gamma_{0}$ is determined by prescribed Cauchy data on $\Gamma_{0}$ in addition to a Dirichlet condition on the known boundary $\Gamma_{1}$. Our main idea is to involve the conformal mapping method as proposed and analyzed by Akduman, Haddar and Kress [2, 9] for the solution of a related inverse boundary value problem. For this we interpret the free boundary $\Gamma_{0}$ as the unknown boundary in the inverse problem to construct $\Gamma_{0}$ from the Dirichlet condition on $\Gamma_{0}$ and Cauchy data on the known boundary $\Gamma_{1}$. Our method for the Bernoulli problem iterates on the missing normal derivative on $\Gamma_{1}$ by alternating between the application of the conformal mapping method for the inverse problem and solving a mixed Dirichlet-Neumann boundary value problem in $D$. We present the mathematical foundations of our algorithm and prove a convergence result. Some numerical examples will serve as proof of concept of our approach.
\end{abstract}

\section{Introduction}

Both free and inverse boundary value problems for partial differential equations are important contemporary topics in applied analysis. Typically in both of them the boundary of the solution domain consists of a known and an unknown part and the latter has to be determined from overdetermined Cauchy data. These Cauchy data are given on the known boundary part in the case of inverse problems and, to the contrary, on the unknown boundary part in the case of free boundary problems. 
A classical example for a free boundary value problem is the Bernoulli problem from fluid dynamics. The velocity field $V$ of an incompressible irrotational fluid flow satisfies $\operatorname{div} V=0$ and $\operatorname{curl} V=0$ and therefore, at least locally, there exists a harmonic function $v$, called velocity potential, such that $V=\operatorname{grad} v$. In two dimensions, in addition to the potential also a stream function $w$ can be introduced as a conjugate harmonic of $v$. Since the velocity $V=\operatorname{grad} v$ is orthogonal to the equipotential lines $v=$ const and since the lines $v=$ const and $w=$ const of the two conjugate harmonic functions are orthogonal, the latter represent the streamlines of the potential flow.

In boundary value problems for such potential flows the boundaries will be either rigid, in which case they are known, or they will be free streamlines, i.e., free boundaries, in which case their shape is not known. On rigid boundaries the fluid flow is assumed to be tangential to the boundary, i.e., the rigid boundary is also a streamline. On a free boundary, to compensate for the fact that its shape is not known, a second boundary condition in addition to $w=$ const has to be imposed. In cases where the free boundary is the interface between the fluid and a surrounding gas the free streamline is in equilibrium with the gas and this means that the pressure $p$ of the fluid at the free streamline is equal to the constant pressure in the gas. Finally conservation of energy, i.e., Bernoulli's law $\frac{1}{2}|V|^{2}+p=$ const allows us to reformulate this condition into the form $|\operatorname{grad} w|=$ const in terms of the stream function $w$. This finally implies that the normal derivative of the stream function $w$ must be constant on the free streamline.

For the concise formulation of the Bernoulli problem we assume that $D_{0}$ and $D_{1}$ are two simply connected bounded domains in $\mathbb{R}^{2}$ with $C^{2}$ smooth boundaries $\Gamma_{0}:=\partial D_{0}$ and $\Gamma_{1}:=\partial D_{1}$ such that $\bar{D}_{1} \subset D_{0}$ and denote by $D$ the doubly connected domain $D:=D_{0} \backslash \bar{D}_{1}$. The unit normal vector $\nu$ to both $\Gamma_{0}$ and $\Gamma_{1}$ is assumed to be directed into the complement of $D$. The Bernoulli free boundary value problem consists in determining the exterior boundary curve $\Gamma_{0}$ such that the unique solution $w \in H^{1}(D)$ to the Dirichlet problem for the Laplace equation

$$
\Delta w=0 \text { in } D
$$

with boundary values

$$
w=0 \quad \text { on } \Gamma_{0}
$$

and

$$
w=1 \quad \text { on } \Gamma_{1}
$$

satisfies

$$
-\frac{\partial w}{\partial \nu}=\lambda \quad \text { on } \Gamma_{0}
$$

where $\Gamma_{1}$ is known and $\lambda$ is a given positive constant. We note that by Hopf's lemma for harmonic functions (see e.g. [6]), the positivity of the constant $\lambda$ is necessary for the existence of a solution to (1.1)-(1.4).

Since the free boundary $\Gamma_{0}$ is the exterior component of $\partial D$, the above problem is referred to as the exterior Bernoulli free boundary value problem. 
Together with the corresponding interior Bernoulli problem where the roles of the free and the known boundary component are interchanged, it has been extensively investigated (see e.g. [1,4] and the literature referenced therein). Existence of a solution has been established by Beurling [5] more than fifty years ago. Contemporary existence proofs are based on variational methods in the sense of shape optimization. Analyticity of the free boundary has been shown by Lewy [14]. If $\Gamma_{1}$ is convex, uniqueness of the solution has been shown by Tepper [15]. Counter examples show that convexity for $\Gamma_{1}$ is necessary for uniqueness (see [7]). Tepper $[15,16]$ has also shown that if $\Gamma_{1}$ is convex or starlike, then so is $\Gamma_{0}$.

Bernoulli's free boundary problem also occurs in electro- and magnetostatics. For example, the optimal design problem to construct an insulation layer that minimizes the current leakage from coaxial cables leads to a Bernoulli problem. A further example is galvanization where in order to produce a layer of constant thickness it is required that the applied electric field has constant strength on the surface of the workpiece. For these examples we refer to [7] and the references therein.

The prominent numerical methods for solving free boundary value problems are so-called trial methods from shape optimization. The basic structure of such methods is to first make an initial guess for the free boundary. Then, firstly, the boundary value problem with one of the conditions on the free boundary omitted is solved and, secondly, the remaining boundary condition is used to update the free boundary. These two steps are iterated until both free boundary conditions are satisfied up to some specified accuracy (see [7, 10, 11] and further references therein).

Here, with the conformal mapping method in mind that was proposed and analyzed by Akduman, Haddar and Kress over the last decade (see [2, 9] among others) we proceed differently and involve the inverse boundary value problem to determine the unknown boundary $\Gamma_{0}$ from the homogeneous Dirichlet condition on $\Gamma_{0}$ and the Cauchy data on $\Gamma_{1}$. For the solution of this inverse problem we introduce the annulus $B$ bounded by two concentric circles $C_{0}$ with radius $\rho>1$ and $C_{1}$ with radius one centered at the origin. In the sequel, we will identify the Euclidean space $\mathbb{R}^{2}$ and the complex plane $\mathbb{C}$ in the usual way. By the Riemann conformal mapping theorem for doubly connected domains there exists a uniquely determined radius $\rho>1$ and a holomorphic function $\Psi$ that maps $B$ bijectively onto $D$ such that the boundaries $C_{0}$ and $C_{1}$ are mapped onto $\Gamma_{0}$ and $\Gamma_{1}$, respectively, with all boundary curves in counter clockwise orientation. The function $\Psi$ is unique up to a rotation of the annulus $B$. We parameterize

$$
\Gamma_{1}=\{\gamma(t): t \in[0,2 \pi)\}
$$

with a continuously differentiable $2 \pi$ periodic function $\gamma: \mathbb{R} \rightarrow \mathbb{C}$ such that $\left.\gamma\right|_{[0,2 \pi)}$ is injective and fix $\Psi$ uniquely $B$ by prescribing $\Psi(1)=\gamma(0)$. Then we define a boundary correspondence function $\varphi:[0,2 \pi] \rightarrow[0,2 \pi]$ by setting

$$
\varphi(t):=\gamma^{-1}\left(\Psi\left(e^{i t}\right)\right), \quad t \in[0,2 \pi] .
$$


Analogous to $D$, the unit normal vector $\nu$ to both $C_{0}$ and $C_{1}$ is directed into the complement of $B$.

The main ingredient of the conformal mapping method is a nonlinear differential equation for the boundary correspondence function $\varphi$ in terms of the given Cauchy data on $\Gamma_{1}$. In the first step of the algorithm this differential equation, in combination with an equation for the radius $\rho$, is solved by successive approximations. Then in the second step, knowing $\rho$ and $\varphi$ a Cauchy problem for $\Psi$ is solved by a Laurent expansion and the unknown boundary is obtained as $\Gamma_{0}=\Psi\left(C_{0}\right)$. Thus we obtain a a parameterization of $\Gamma_{0}$ by

$$
\chi(t):=\Psi\left(\rho e^{i t}\right), \quad t \in[0,2 \pi] .
$$

For the purpose of iteratively solving the Bernoulli problem, we make an initial guess for the unknown normal derivative $g:=\partial_{\nu} w$ on $\Gamma_{1}$ and apply our conformal mapping method to construct an approximation for the unknown $\Gamma_{0}$. Then we update $g$ by the normal derivative on $\Gamma_{1}$ of the solution to the mixed Dirichlet-Neumann problem (1.2) and (1.4) and iterate these two steps. We will present the mathematical foundation of this algorithm and prove a convergence result. Numerical examples will confirm the feasibility of the method.

The plan of the paper is as follows. In Section 2 we will outline the basic properties of the conformal mapping algorithm for the inverse boundary value problem as required for our purpose. Then we will present the details of our proposed algorithm for the free boundary value problem in Section 3 followed by a convergence analysis in Section 4. The numerical examples in Section 5 serve as proof of concept.

To conclude this introduction we briefly sketch a further possibility to solve the Bernoulli problem with the aid of the conformal mapping $\Psi$. For this we note that the solution $v:=w \circ \Psi$ to the Dirichlet problem in $B$ with boundary values $v=1$ on $C_{1}$ and $v=0$ on $C_{0}$ is given by

$$
v(x)=1-\frac{\ln |x|}{\ln \rho}, \quad x \in B,
$$

and has normal derivatives

$$
\frac{\partial v}{\partial \nu}=-\frac{1}{\rho \ln \rho} \quad \text { on } C_{0}
$$

and

$$
\frac{\partial v}{\partial \nu}=\frac{1}{\ln \rho} \quad \text { on } C_{1} .
$$

We denote by $\widetilde{w}$ a conjugate harmonic of $w$ and set $\widetilde{v}=\widetilde{w} \circ \Psi$, that is, $\widetilde{v}$ is a conjugate harmonic of $v$. Then in view of (1.4) and (1.8) from the CauchyRiemann equations, both for $w$ and $\widetilde{w}$ and for $v$ and $\widetilde{v}$ we obtain that

$$
\begin{aligned}
-\lambda\left|\chi^{\prime}(t)\right| & =\left|\chi^{\prime}(t)\right| \frac{\partial w}{\partial \nu}(\chi(t))=\left|\chi^{\prime}(t)\right| \frac{\partial \widetilde{w}}{\partial s}(\chi(t))=\frac{d}{d t} \widetilde{w}(\chi(t)) \\
& =\frac{d}{d t} \widetilde{v}\left(\rho e^{i t}\right)=\rho \frac{\partial v}{\partial \nu}\left(\rho e^{i t}\right)=-\frac{1}{\ln \rho}
\end{aligned}
$$


for $0 \leq t \leq 2 \pi$, that is,

$$
\left|\chi^{\prime}(t)\right|=\frac{1}{\lambda \ln \rho}, \quad 0 \leq t \leq 2 \pi .
$$

By Cauchy's integral formula, we can represent the holomorphic function $\Psi$ by its boundary values $\chi$ and $\varphi$ as

$$
\Psi(z)=\frac{\rho}{2 \pi} \int_{0}^{2 \pi} \frac{\chi(\tau) e^{i \tau}}{\rho e^{i \tau}-z} d \tau-\frac{1}{2 \pi} \int_{0}^{2 \pi} \frac{\gamma(\varphi(\tau)) e^{i \tau}}{e^{i \tau}-z} d \tau, \quad z \in B .
$$

From this, by the Sokhotski-Plemelj jump relations (see [13]) for the Cauchy integral by letting $z$ tend to $C_{0}$ and $C_{1}$ we obtain the two integral equations

$$
\chi(t)=\frac{1}{2 \pi} \int_{0}^{2 \pi} \frac{\chi(\tau) e^{i \tau}}{e^{i \tau}-e^{i t}} d \tau-\frac{1}{2 \pi} \int_{0}^{2 \pi} \frac{\gamma(\varphi(\tau)) e^{i \tau}}{e^{i \tau}-\rho e^{i t}} d \tau, \quad 0 \leq t \leq 2 \pi,
$$

and

$$
\gamma(\varphi(t))=\frac{\rho}{2 \pi} \int_{0}^{2 \pi} \frac{\chi(\tau) e^{i \tau}}{\rho e^{i \tau}-e^{i t}} d \tau-\frac{1}{2 \pi} \int_{0}^{2 \pi} \frac{\gamma(\varphi(\tau)) e^{i \tau}}{e^{i \tau}-e^{i t}} d \tau, \quad 0 \leq t \leq 2 \pi,
$$

for $\chi$ and $\varphi$. In our future research on the Bernoulli problem we intend to explore the possibility to solve these two nonlinear integral equations using the additional condition (1.10).

\section{The conformal mapping method adjusted}

We note that as opposed to our previous papers on the conformal mapping method the unknown boundary $\Gamma_{0}$ is the exterior part of the boundary $\partial D$. With this reversion of the roles of the exterior and interior boundaries the conformal method solves the inverse boundary value problem to find the unknown boundary $\Gamma_{0}$ from the Cauchy data $f=\left.u\right|_{\Gamma_{1}}$ and $g=\left.\partial_{\nu} u\right|_{\Gamma_{1}}$ on $\Gamma_{1}$ of a harmonic function $u \in H^{1}(D)$ satisfying $u=0$ on $\Gamma_{0}$. We proceed by a brief adjustment of the conformal mapping method for this special case.

Proceeding analogous to the derivation of (1.10), from the Neumann conditions $\partial_{\nu} u=g$ on $\Gamma_{1}$ and (1.9) we obtain that

$$
g(\gamma(\varphi(t)))\left|\frac{d}{d t} \gamma(\varphi(t))\right|=\frac{1}{\ln \rho}
$$

for $0 \leq t \leq 2 \pi$. Since the boundary correspondence function $\varphi$ is strictly monotonically increasing, this implies

$$
\varphi^{\prime}=\frac{1}{\ln \rho\left|\gamma^{\prime} \circ \varphi\right| g \circ \gamma \circ \varphi}
$$

together with the boundary conditions

$$
\varphi(0)=0, \quad \varphi(2 \pi)=2 \pi .
$$


Integrating (2.1) over the interval $[0,2 \pi]$ we obtain

$$
\frac{1}{\ln \rho}=\frac{1}{2 \pi} \int_{\Gamma_{1}} g d s
$$

as formula for the radius $\rho$. We note that in the general case for arbitrary Dirichlet data $f$ the formula for the radius corresponding to (2.4) contains the unknown $\varphi$ and the differential equation corresponding to $(2.2)$ is nonlocal (see $[2,9]$ ).

To deal with the two boundary conditions (2.3) for the differential equation of order one, integrating (2.2) we obtain

$$
\frac{1}{2 \pi} \int_{0}^{2 \pi} \frac{1}{\ln \rho\left|\gamma^{\prime} \circ \varphi\right| g \circ \gamma \circ \varphi} d t-1=0 .
$$

Subtracting this from (2.2) and inserting (2.4) for $\rho$ leads to the modified differential equation

$$
\varphi^{\prime}=\frac{\int_{\Gamma_{1}} g d s}{2 \pi\left|\gamma^{\prime} \circ \varphi\right| g \circ \gamma \circ \varphi}-\frac{\int_{\Gamma_{1}} g d s}{4 \pi^{2}} \int_{0}^{2 \pi} \frac{1}{\left|\gamma^{\prime} \circ \varphi\right| g \circ \gamma \circ \varphi} d t+1
$$

that the boundary correspondence function $\varphi$ has to satisfy together with the initial condition

$$
\varphi(0)=0
$$

Obviously, each solution $\varphi$ to the initial value problem (2.5)-(2.6) automatically also satisfies the second boundary condition $\varphi(2 \pi)=2 \pi$.

Provided $g$ is positive on all of $\Gamma_{1}$, the classical Picard-Lindelöf theorem guarantees uniqueness and existence for the initial value problem (2.5)-(2.6) and this solution can be obtained by successive approximations. By Hopf's lemma combined with the maximum-minimum principle, the required positivity is satisfyed for the Neumann trace $g=\left.\partial_{\nu} u\right|_{\Gamma_{1}}$ of a harmonic function in $H^{1}(D)$ satisfying $u=1$ on $\Gamma_{1}$ and $-\partial_{\nu} u=\lambda$ on $\Gamma_{0}$.

From the equivalent form of the initial value problem (2.5)-(2.6) as a nonlinear Volterra integral equation, by the implicit function theorem it can be seen that the operator $T: H^{1}\left(\Gamma_{1}\right) \rightarrow H^{1}[0,2 \pi]$ given by

$$
T: g \mapsto \varphi
$$

which maps the Neumann data $g$ onto the solution $\varphi$ of $(2.5)-(2.6)$ is Fréchet differentiable and that the Fréchet derivative $d T(g ; h)$ of $T$ at $g$ in the direction $h$ is given by the unique solution to the linear differential equation

$$
[d T(g ; h)]^{\prime}=\alpha(g) d T(g ; h)+\beta(g ; h)-\frac{1}{2 \pi} \int_{0}^{2 \pi}\{\alpha(g) d T(g ; h)+\beta(g ; h)\} d t
$$

satisfying the initial condition

$$
d T(g ; h)(0)=0 .
$$


Here we abbreviated

$$
\alpha(g):=-\frac{\left(\gamma^{\prime} \circ \varphi\right) \cdot\left(\gamma^{\prime \prime} \circ \varphi\right) \int_{\Gamma_{1}} g d s}{2 \pi\left|\gamma^{\prime} \circ \varphi\right|^{3} g \circ \gamma \circ \varphi}-\frac{(g \circ \gamma)^{\prime} \circ \varphi \int_{\Gamma_{1}} g d s}{2 \pi\left|\gamma^{\prime} \circ \varphi\right|[g \circ \gamma \circ \varphi]^{2}}
$$

and

$$
\beta(g ; h):=-\frac{h \circ \gamma \circ \varphi \int_{\Gamma_{1}} g d s}{2 \pi\left|\gamma^{\prime} \circ \varphi\right|[g \circ \gamma \circ \varphi]^{2}}+\frac{\int_{\Gamma_{1}} h d s}{2 \pi\left|\gamma^{\prime} \circ \varphi\right| g \circ \gamma \circ \varphi}
$$

with $\varphi=T g$.

For the Cauchy problem part of the conformal mapping method we expand

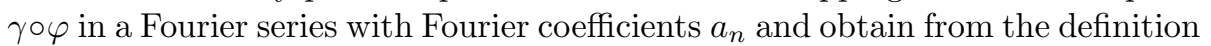
(1.5) of $\varphi$ that

$$
\Psi\left(e^{i t}\right)=\sum_{n=-\infty}^{\infty} a_{n} e^{i n t}, \quad t \in[0,2 \pi] .
$$

By the Laurent series for $\Psi$, in view of (1.6), this implies

$$
\chi(t):=\sum_{n=-\infty}^{\infty} a_{n} \rho^{n} e^{i n t}, \quad t \in[0,2 \pi]
$$

for the parametrization of $\Gamma_{0}$. In order to numerically cope with the instability of this parametrization with respect to numerical errors in the Fourier coefficients $a_{n}$ for $n>0$ we incorporate a regularization by truncating the series in the form

$$
\chi_{N}(t):=\sum_{n=-N+1}^{N+1} a_{n} \rho^{n} e^{i n t}, \quad t \in[0,2 \pi],
$$

where $N \in \mathbb{N}$ serves as a regularization parameter. This may be interpreted as a singular value cut-off. We note that also a Tikhonov type regularization can be used (see $[2,9]$ ).

We denote by $R, R_{N}: H^{1}[0,2 \pi] \rightarrow C^{2}[0,2 \pi]$ the linear operators that map the series (2.11) onto the series (2.12) and (2.13) for $\chi$ and $\chi_{N}$, respectively. Then we can summarize the conformal mapping method by the operators

$$
M:=R \gamma T \quad \text { and } \quad M_{N}=R_{N} \gamma T
$$

from $H^{1}\left(\Gamma_{1}\right)$ into $C^{2}[0,2 \pi]$ that map the given normal derivative $g$ onto the parameterizations $\chi$ and $\chi_{N}$, respectively. By the chain rule, the Fréchet derivative of $M$ is given by

$$
d M(g ; h):=R\left[\gamma^{\prime} \circ(T g) d T(g ; h)\right]-2 \pi \rho \frac{\int_{\Gamma_{1}} h d s}{\left[\int_{\Gamma_{1}} g d s\right]^{2}} R^{\prime}(\gamma \circ T g),
$$

where $R^{\prime}$ denotes the derivative of the series (2.12) with respect to $\rho$. For $d M_{N}(g ; h)$ we have an analogous expression with $R$ replaced by $R_{N}$. 


\section{The free boundary problem algorithm}

The main idea of our iterative method for the free boundary value problem is to apply the conformal mapping algorithm of the previous section with the Cauchy pair $f=1$ and $g=\left.w\right|_{\Gamma_{1}}$ for the solution of (1.1)-(1.3). For this we make an initial guess for the unknown normal derivative $g$ and then iterate by alternating between the conformal mapping method for obtaining an approximation for $\Gamma_{0}$ and updating $g$ by solving the mixed Dirichlet-Neumann problem (1.1), (1.2) and (1.4).

\section{The Algorithm}

1 Choose an initial guess $g$.

2 Apply the conformal mapping method to obtain an approximation $\Gamma_{0}$ by (2.12), or for the sake of stability by (2.13).

3 Solve the mixed Dirichlet-Neumann problem for $\Delta w=0$ in $D$ with boundary conditions

$$
w=1 \quad \text { on } \Gamma_{1}
$$

and

$$
-\frac{\partial w}{\partial \nu}=\lambda \quad \text { on } \Gamma_{0}
$$

by a direct integral equation method, i.e., an integral equation for the unknowns $\left.\partial_{\nu} w\right|_{\Gamma_{1}}$ and $\left.w\right|_{\Gamma_{0}}$.

If $\left\|g-\left.\partial_{\nu} w\right|_{\Gamma_{1}}\right\|_{\infty}<\delta$ for a given tolerance $\delta$ terminate the iteration. Otherwise update $g:=\left.\partial_{\nu} w\right|_{\Gamma_{1}}$ and go back to Step 2.

After introducing the operator $F: C^{2}[0,2 \pi] \rightarrow H^{1}\left(\Gamma_{1}\right)$ that maps the parameterization $\chi$ of $\Gamma_{0}$ onto the normal derivative $g$ of the solution to (3.1)-(3.2) on $\Gamma_{1}$ we note that the iteration scheme without regularization in the second step can be expressed in terms of the operator

$$
A:=F M
$$

as

$$
g_{n+1}=A g_{n}, \quad n=0,1,2, \ldots
$$

Later on we will find in necessary for convergence to modify (3.4) by applying a relaxation

$$
g_{n+1}=\omega g_{n}+(1-\omega) A g_{n}, \quad n=0,1,2, \ldots,
$$

with a relaxation parameter $\omega \in[0,1)$ adjusted to the value of $\lambda$ for the Neumann boundary condition on $\Gamma_{1}$.

In Step 3 alternatively one could solve the corresponding Dirichlet-Neumann problem in the annulus $B$ via Fourier series. However, this leads to an update for $g \circ \varphi$ whereas Step 2 requires $g$ on $\Gamma_{1}$ in order to obtain an update for $\varphi$ 
via the iteration step for (2.5)-(2.6). Using the boundary integral equation in $D$ itself avoids the issue of a numerical inversion of $\varphi$ to obtain $\left.g\right|_{\Gamma_{1}}$ from $g \circ \varphi$.

In principle, for Step 2 the conformal mapping method could be replaced by any other solution method for the inverse boundary value problem. We have chosen the conformal mapping method for two reasons. Firstly, complex analysis methods have played an important role in the history of free boundary problems for the Laplace equation (see e.g. [8]). Secondly, and more importantly, for this particular choice for Step 2 we were able to analyze the convergence based on our techniques from the conformal mapping method for the inverse problem.

For completeness we briefly outline the use of boundary integral equations for the solution of (3.1)-(3.2). In terms of the fundamental solution

$$
\Phi(x, y):=\frac{1}{2 \pi} \ln \frac{1}{|x-y|}, \quad x \neq y,
$$

to the Laplace equation, Green's integral formula applied to a solution $w$ of (3.1)-(3.2) reads

$$
w(x)=\int_{\Gamma_{1}} \Phi(x, \cdot) g d s-\int_{\Gamma_{0}}\left\{\lambda \Phi(x, \cdot)+\frac{\partial \Phi(x, \cdot)}{\partial \nu} \omega\right\} d s
$$

for $x \in D$ where we have set $g:=\left.\partial \nu w\right|_{\Gamma_{1}}$ and $\omega:=\left.w\right|_{\Gamma_{0}}$ and used the fact that the double-layer potential with constant density on $\Gamma_{1}$ vanishes in $D$. Letting $x$ approach $\Gamma_{0}$ and $\Gamma_{1}$ we obtain the system of two integral equations

$$
\frac{\omega}{2}+\int_{\Gamma_{0}} \frac{\partial \Phi(x, \cdot)}{\partial \nu} \omega d s-\int_{\Gamma_{1}} \Phi(x, \cdot) g d s=-\lambda \int_{\Gamma_{0}} \Phi(x, \cdot) d s, \quad x \in \Gamma_{0},
$$

and

$$
\int_{\Gamma_{0}} \frac{\partial \Phi(x, \cdot)}{\partial \nu} \omega d s-\int_{\Gamma_{1}} \Phi(x, \cdot) g d s=-\lambda \int_{\Gamma_{0}} \Phi(x, \cdot) d s-1, \quad x \in \Gamma_{1},
$$

for the two unknowns $\omega$ and $g$.

Without loss of generality, we assume that there exists a point $z$ in $D_{1}$ such that $|x-z| \neq 1$ for all $x \in D_{1}$. Then Theorem 3.16 in [12] (see also [13, Theorem 7.38]) guarantees that the single-layer operator from $H^{-1 / 2}\left(\Gamma_{1}\right)$ into $H^{1 / 2}\left(\Gamma_{1}\right)$ is bijective. Note that by a change of variables the setting always can be scaled such that this condition is satisfied. For simplicity, we have chosen to just work with the single-layer operator and point out that alternatively in order to obtain injectivity we could modify it by adding an additional term as in [13, Theorem 7.41].

We note that plugging in $\omega=0$ for the zero Dirichlet condition on the free boundary makes (3.7)-(3.8) a system of integral equations for the unknown free boundary $\Gamma_{0}$ and the unknown normal derivative $\left.\partial_{\nu} w\right|_{\Gamma_{1}}$ as a slip variable. With this interpretation, the (3.7)-(3.8) serves as basis for an integral equation method for the solution of the Bernoulli problem due to Trefftz [17, 18].

Using the jump relations and taking care of the logarithmic behavior of the single-layer potentials as in the proof of Theorem 7.38 in [13] it can be shown 
that the homogeneous form of (3.7)-(3.8) only has the trivial solution and that for any solution $\omega$ and $g$ of the inhomogeneous equation the function $w$ defined in $D$ by the right hand side of (3.6) provides a solution of (3.1)-(3.2). Finally, after the regularization of equation (3.8) with the inverse of the single-layer operator on $\Gamma_{1}$ the Riesz theory for compact operators can be employed to show existence of a unique solution $\omega \in H^{1 / 2}\left(\Gamma_{0}\right)$ and $g \in H^{-1 / 2}\left(\Gamma_{1}\right)$. Using the regularity of the right hand side and the mapping properties of the involved boundary operators higher regularity of the solution can be achieved.

For the approximate solution of (3.7)-(3.8) we recommend the efficient Nyström and collocation method for boundary integral equations for harmonic functions based on trigonometric approximations as described in [13].

\section{A convergence result}

We begin our considerations on the convergence of the above iteration scheme by some preliminary observations on the simple case when $\Gamma_{1}$ is the unit circle. In this case the solution to the Bernoulli free boundary problem is given by a concentric circle $\Gamma_{0}$ and from (1.7) we observe that its radius is given by the nonlinear equation

$$
\frac{1}{\rho \ln \rho}=\lambda
$$

and that

$$
g=\frac{1}{\ln \rho} .
$$

We start the iteration with a constant and assume the $n$-th iteration to be given by a constant $g_{n}$. Then from (2.4) we have

$$
\rho_{n}=\exp \left(\frac{1}{g_{n}}\right)
$$

and the solution in Step 3 becomes $w(x)=1-\rho_{n} \lambda \ln |x|$. Hence, the update equation is given by

$$
g_{n+1}=\lambda \exp \left(\frac{1}{g_{n}}\right) .
$$

The function $q: t \mapsto \lambda \exp (1 / t)$ has derivative $q^{\prime}(1 / \ln \rho)=-\ln \rho$. Therefore, provided $g>1$, that is, $\rho<e$, the iterations (4.3) converge locally, i.e., we have convergence if the initial guess is not too far from the correct $g$. From (4.1) we observe that $\rho<e$ is satisfied if $\lambda>e^{-1}$, since clearly $\rho>e$ implies that $\lambda<e^{-1}$.

For a more general convergence result, in principle, we need to estimate the norm of the derivative of the iteration operator $A$ as given by (3.3) via

$$
d A(g ; h)=d F(M g ; d M(g ; h)) .
$$

Therefore, in addition to the Fréchet derivative of $M$ given by (2.7) and (2.14) we also need the Fréchet derivative of $F$. According to Allaire [3, Corollary 6.36] 
the Fréchet derivative of $F$ at $\chi$ in the direction $\eta$ is given by $d F(\chi ; \eta)=\left.\partial_{\nu} v\right|_{\Gamma_{1}}$ where $v$ is the unique solution to the mixed Dirichlet-Neumann problem in $D$ with boundary condition

$$
v=0 \quad \text { on } \Gamma_{1}
$$

and

$$
\frac{\partial v}{\partial \nu}=-\kappa \lambda \nu \cdot \eta+\frac{\partial}{\partial s} \nu \cdot \eta \frac{\partial w}{\partial s} \quad \text { on } \Gamma_{0}
$$

in terms of the solution $w$ of (3.1)-(3.2). Here, $s$ is the arc length on $\Gamma_{0}$ and $\kappa$ denotes the curvature of $\Gamma_{0}$.

Since deriving sharp estimates on the norm of $d A$ based on the representations of $d M$ and $d F$ appears out of reach in the general case, we confine ourselves to the simple situation when $\Gamma_{1}$ is the unit circle with the canonical parameterization $\gamma(t)=e^{i t}$, that is, when $D=B$. In this case $g$ is a constant and the exact values for $\rho$ and $g$ are given by (4.1) and (4.2). We proceed by evaluating the Fréchet derivative at the exact solution $g$ and consequently also the exact $\varphi$ given by $\varphi(t)=t$. Since $\gamma^{\prime} \cdot \gamma^{\prime \prime}=0$ and $g^{\prime}=0$ from (2.9) we obtain that $\alpha(g)=0$. From (2.10), using (4.2), we find that

$$
\beta(g ; h)=\ln \rho\left(-h \circ \gamma \circ \varphi+\frac{1}{2 \pi} \int_{\Gamma_{1}} h d s\right) .
$$

With this, solving (2.7) and (2.8) for $h=1$ leads to

$$
d T(g ; 1)=0
$$

and from (2.14) we obtain

$$
d M(g ; 1)(t)=-\rho(\ln \rho)^{2} e^{i t} .
$$

Solving (2.7) and (2.8) for the trigonometric monomials $c_{n}(t):=\cos n t$ and $s_{n}(t):=\sin n t$ for $n \in \mathbb{N}$ we obtain

$$
d T\left(g ; c_{n} \circ \gamma^{-1}\right)=-\frac{\ln \rho}{n} s_{n} \quad \text { and } \quad d T\left(g ; s_{n} \circ \gamma^{-1}\right)=\frac{\ln \rho}{n}\left(c_{n}-1\right)
$$

whence

$$
d M\left(g ; c_{n} \circ \gamma^{-1}\right)(t)=\frac{\ln \rho}{2 n}\left\{\rho^{1-n} e^{(1-n) i t}-\rho^{1+n} e^{(1+n) i t}\right\}
$$

and

$$
d M\left(g ; s_{n} \circ \gamma^{-1}\right)(t)=\frac{i \ln \rho}{2 n}\left\{\rho^{1-n} e^{(1-n) i t}+\rho^{1+n} e^{(1+n) i t}-2 \rho e^{i t}\right\}
$$

follow by (2.12) and (2.14).

Now we need to consider the mixed Dirichlet-Neumann problem (4.5)-(4.6) in $D$ for $\eta$ given by the right hand sides of (4.8) and (4.9) and interpreted as a vector in $\mathbb{R}^{2}$ for the inner product with the normal vector $\nu$ on $\Gamma_{0}$. Since the 
solution $w$ of (3.1)-(3.2) is constant and $\kappa \lambda=\lambda / \rho=1 / \rho^{2} \ln \rho$, the Neumann condition (4.6) reduces to

$$
\frac{\partial v}{\partial \nu}=-\frac{1}{\rho^{2} \ln \rho} \nu \cdot \eta \quad \text { on } \Gamma_{0} .
$$

This becomes

$$
\frac{\partial v}{\partial \nu}=\frac{\ln \rho}{\rho} \quad \text { on } \Gamma_{0}
$$

for $\eta=d M(g ; 1)$,

$$
\frac{\partial v}{\partial \nu}\left(\rho e^{i t}\right)=\frac{1}{2 n \rho}\left(\rho^{-n}-\rho^{n}\right) \cos n t, \quad t \in[0,2 \pi],
$$

for $\eta=d M\left(g ; c_{n} \circ \gamma^{-1}\right)$, and

$$
\frac{\partial v}{\partial \nu}\left(\rho e^{i t}\right)=\frac{1}{2 n \rho}\left(\rho^{-n}-\rho^{n}\right) \sin n t, \quad t \in[0,2 \pi],
$$

for $\eta=d M\left(g ; s_{n} \circ \gamma^{-1}\right)$. The correponding solutions to (4.5)-(4.6) are

$$
v(x)=\ln \rho \ln |x|, \quad 1 \leq|x| \leq \rho,
$$

for $h=1$,

$$
v\left(r e^{i t}\right)=-\frac{1}{2 n^{2}} \frac{\rho^{2 n}-1}{\rho^{2 n}+1}\left(r^{n}-r^{-n}\right) \cos n t, \quad 1 \leq r \leq \rho, t \in[0,2 \pi],
$$

for $h=c_{n} \circ \gamma^{-1}$ and

$$
v\left(r e^{i t}\right)=-\frac{1}{2 n^{2}} \frac{\rho^{2 n}-1}{\rho^{2 n}+1}\left(r^{n}-r^{-n}\right) \sin n t, \quad 1 \leq r \leq \rho, t \in[0,2 \pi],
$$

for $h=s_{n} \circ \gamma^{-1}$. Its normal derivatives on $\Gamma_{1}$ become

$$
\frac{\partial v}{\partial \nu}=-\ln \rho \quad \text { on } \Gamma_{1}
$$

for $h=1$,

$$
\frac{\partial v}{\partial \nu}\left(e^{i t}\right)=\frac{1}{n} \frac{\rho^{2 n}-1}{\rho^{2 n}+1} \cos n t, \quad t \in[0,2 \pi],
$$

for $h=c_{n} \circ \gamma^{-1}$, and

$$
\frac{\partial v}{\partial \nu}\left(e^{i t}\right)=\frac{1}{n} \frac{\rho^{2 n}-1}{\rho^{2 n}+1} \sin n t, \quad t \in[0,2 \pi],
$$

for $h=s_{n} \circ \gamma^{-1}$. In view of (4.4) and $M g=\rho \gamma$, this finally implies that

$$
d A(g ; 1)=\mu_{0}
$$


with the eigenvalue

$$
\mu_{0}=-\ln \rho,
$$

a result that we also can obtain more easily from (4.3), and further

$$
\left.d A\left(g ; c_{n} \circ \gamma^{-1}\right)\right)=\mu_{n} c_{n} \circ \gamma^{-1}
$$

and

$$
\left.d A\left(g ; s_{n} \circ \gamma^{-1}\right)\right)=\mu_{n} s_{n} \circ \gamma^{-1}
$$

with the eigenvalues

$$
\mu_{n}=\frac{1}{n} \frac{\rho^{2 n}-1}{\rho^{2 n}+1}
$$

for $n \in \mathbb{N}$.

For $n \geq 2$ consider the function $p:[1, \infty) \rightarrow \mathbb{R}$ defined by

$$
p(t):=n\left(t^{n}+1\right)-\frac{t+1}{t-1}\left(t^{n}-1\right) .
$$

With the aid of a geometric sum it can be seen that

$$
p(t)=n\left(t^{n}+1\right)-1-2 \sum_{k=1}^{n-1} t^{k}-t^{n}
$$

and for $t \geq 1$ this implies

$$
p^{\prime}(t)=n(n-1) t^{n-1}-2 \sum_{k=1}^{n-1} k t^{k-1} \geq n(n-1) t^{n-1}-2 t^{n-1} \sum_{k=1}^{n-1} k \geq 0 .
$$

Therefore $p$ is nonnegative on $[0, \infty)$ since $p(1)=0$. Setting $t=\rho^{2}$, from this we now can deduce that

$$
\sup _{n \in \mathbb{N}} \mu_{n}=\mu_{1} .
$$

From the above eigenfunctions and eigenvalues we now can conclude that for $\Gamma_{1}$ a circle the Fréchet derivative $d A(g ; \cdot): H^{1}\left(\Gamma_{1}\right) \rightarrow H^{1}\left(\Gamma_{1}\right)$ at the exact $g$ is a self adjoint compact operator with spectral radius

$$
\sigma=\max \left(\ln \rho, \frac{\rho^{2}-1}{\rho^{2}+1}\right)
$$

which is less than one if $\rho<e$. As already observed above, $\lambda>e^{-1}$ implies that $\rho<e$ as consequence of (4.1).

If we consider the regularized iteration operator $A_{N}$ where $R$ is replaced by the truncated Cauchy operator $R_{N}$, then $\mu_{n}, n=0,1,2, \ldots, N$, are also eigenvalues of $d A_{N}(g ; \cdot)$ and the remaining eigenvalues become zero. Hence, the norms of $d A_{N}(g ; \cdot)$ and $d A(g ; \cdot)$ coincide.

From the presentation of the Fréchet derivative of $A$ we can observe that all its components except the ill-posed Cauchy operator $R$ depend continuously on 
$g$ and $\Gamma_{1}$. Hence, the Fréchet derivative of the regularized operator $A_{N}$ depends continuously on $g$ and $\Gamma_{1}$. Therefore, for fixed $N$, if $\Gamma_{1}$ is sufficiently close to a circle, there exists a ball centered at the correct $g$ such that within this ball the Fréchet derivative of $A_{N}$ has norm less than one. Therefore, making use of the mean value theorem for nonlinear operators, the following theorem can be proven.

Theorem 4.1 Assume that $\Gamma_{1}$ is sufficiently close to a circle and $\lambda>e^{-1}$. Then the iterations $g_{n+1}=A_{N} g_{n}, n=0,1,2, \ldots$, converge provided the initial guess $g_{0}$ is sufficiently close to the exact normal derivative $g$ for the circle.

The eigenvalues $\omega+(1-\omega) \mu_{n}, n \in N$, of the Fréchet derivative of the relaxation operator $B(\omega):=\omega I+(1-\omega) A$ (for the circle at the exact solution) has absolute value smaller than one for $\omega \in[0,1)$ and, depending on $\lambda$, the parameter $\omega$ can be chosen such that $\omega-(1-\omega) \ln \rho$ also has absolute value smaller than one. Therefore the restriction $\lambda>e^{-1}$ of our theorem is not essential, since it can be circumvented by the relaxation iterations $g_{n+1}:=$ $B(\omega) g_{n}$.

As we will illustrate by the numerical examples in the following final section, convergence also occurs for boundaries $\Gamma_{1}$ with shapes quite different from circles.

\section{Numerical examples}

As proof of concept rather than a documentation of a fully developed code, in this final section we present some numerical constructions for free boundaries. For the known rigid boundary $\Gamma_{1}$ we choose the parameterization

$$
\gamma(t)=(1.5 \cos t+\varepsilon \cos 2 t, 2 \sin t), \quad 0 \leq t \leq 2 \pi,
$$

which satisfies our geometric assumption that ensures invertibility of the singlelayer operator. For the shape parameter $\varepsilon$ in (5.1) and the parameter $\lambda$ for the Neumann condition we have chosen the values $\varepsilon=0.2,0.4,0.6$ and $\lambda=0.5,2$. In the figures the rigid boundary $\Gamma_{1}$ is given as dotted (green) curve and the free boundary $\Gamma_{0}$ as full (red) curve.

For the solution of the initial value problem (2.5)-(2.6) we approximated the iterations $\varphi_{n}$ by a trigonometric polynomial of degree $N$ in the form

$$
\varphi_{n}(t)=t+\sum_{k=0}^{N} \alpha_{k, n} \cos k t+\sum_{k=1}^{N} \beta_{k, n} \sin k t .
$$

We collocated

$$
\varphi_{n+1}^{\prime}=\frac{\int_{\Gamma_{1}} g d s}{2 \pi\left|\gamma^{\prime} \circ \varphi_{n}\right| g \circ \gamma \circ \varphi_{n}}-\frac{\int_{\Gamma_{1}} g d s}{4 \pi^{2}} \int_{0}^{2 \pi} \frac{1}{\left|\gamma^{\prime} \circ \varphi_{n}\right| g \circ \gamma \circ \varphi_{n}} d t+1
$$

at the $M$ equidistant points $t_{m}:=2 \pi m / M, m=1,2, \ldots, M$, to obtain a linear system of $M+1$ equations, including the initial condition, for the $2 N+1$ Fourier 
coefficients of $\varphi_{n+1}$ which we solved by least squares. We used trigonometric polynomials of degree $N=8$ and $M=62$ collocation points and kept the number $N$ also for the truncation of the Laurent series. For the number $Z_{i}$ of inner iterations to solve (2.5)-(2.6) we made the a priori choice $Z_{i}=5$. The iterations where started with $g_{0}=2 \lambda$ resulting in $\varphi_{0}(t)=t$.

For the solution of the Dirichlet-Neumann problem we numerically solved the integral equations (3.7)-(3.8) by Nyström and collocation methods based on trigonometric polynomial interpolation as described in [13]. We again used $M=64$ equidistant collocation points for each of the two equations. The results did not change significantly for $M=128$ collocation points.

For the outer iterations we used the relaxation factor $\omega=0.5$ and they were stopped when the normal derivative on $\Gamma_{1}$ changed less than $\delta=0.00001$ in the maximum norm.
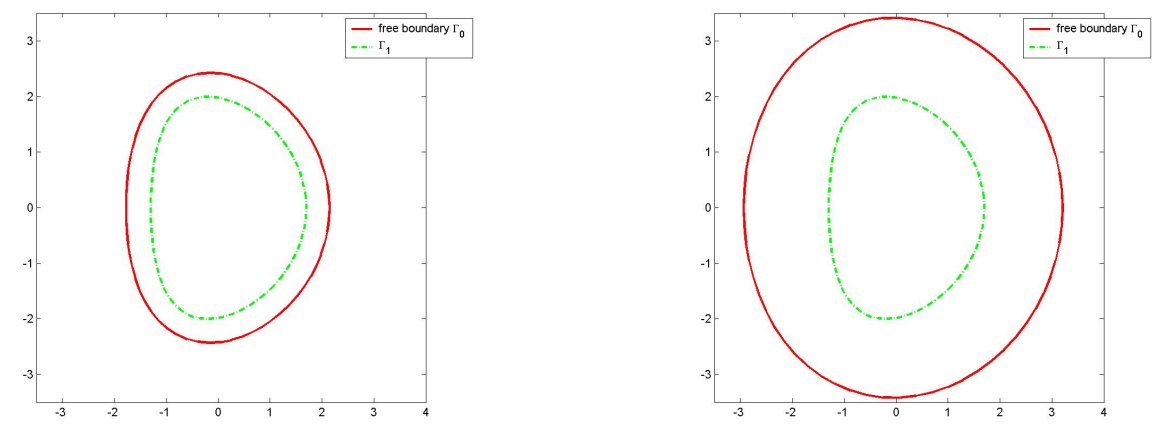

Figure 5.1: Free boundary for $\varepsilon=0.2$ and $\lambda=2$ (left) and $\lambda=0.5$ (right)
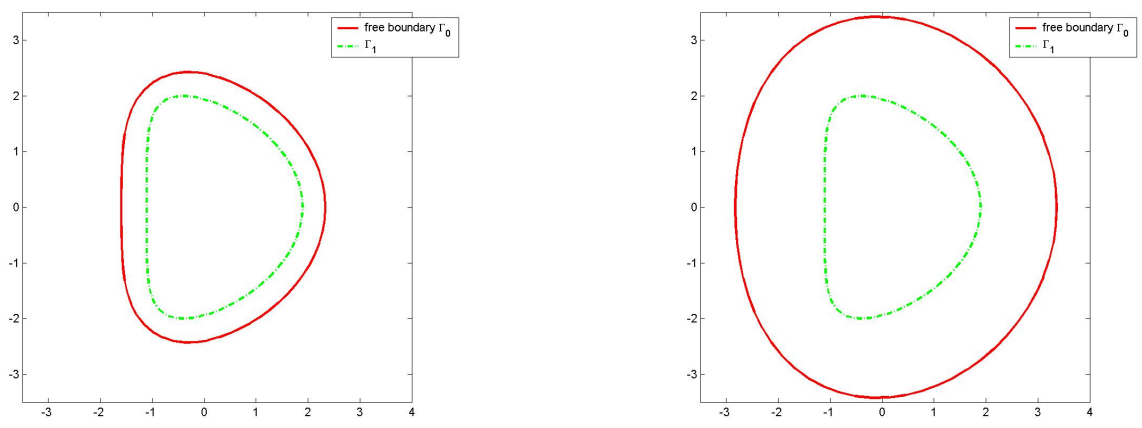

Figure 5.2: Free boundary for $\varepsilon=0.4$ and $\lambda=2$ (left) and $\lambda=0.5$ (right)

Table 5.1 gives the required number $Z_{o}$ of outer iterations together with the final value $\|w\|_{\infty}$ of the maximum norm of the Dirichlet values on the free boundary. 

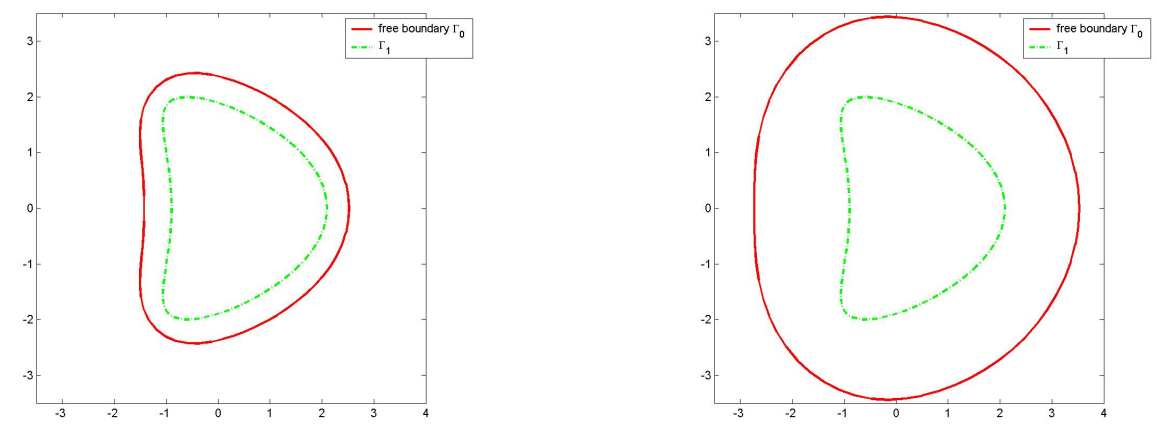

Figure 5.3: Free boundary for $\varepsilon=0.6$ and $\lambda=2$ (left) and $\lambda=0.5$ (right)

\begin{tabular}{|c|r|c|c|}
\hline$\varepsilon$ & $\lambda$ & $Z_{o}$ & $\|w\|_{\infty}$ \\
\hline & & & \\
0.2 & 0.5 & 12 & 0.00003 \\
0.2 & 2 & 17 & 0.00092 \\
0.4 & 0.5 & 13 & 0.00016 \\
0.4 & 2 & 19 & 0.00350 \\
0.6 & 0.5 & 16 & 0.00881 \\
0.6 & 2 & 21 & 0.00671 \\
\hline
\end{tabular}

Table 5.1: Iteration numbers and final Dirichlet norm

The increase of the deviation form the zero Dirichlet condition on the free boundary for geometries with larger deviations from an circular annulus, obviously, is due to the fact that our algorithm does not impose this Dirichlet condition explicitly. Nevertheless, the examples indicate satisfactory performance of our algorithm although we violated the principle not to destroy stability of a problem by incorporating an ill-posed component in its numerical solution.

This research was initiated while R.K. was visiting INRIA Saclay Ile de France and Ecole Polytechnique, Palaiseau. The hospitality and the support are gratefully acknowledged.

\section{References}

[1] Acker, A.: On the geometric form of Bernoulli configurations. Math. Meth. Appl. Sci. 10, 1-14 (1988).

[2] Akduman, I. and Kress, R.: Electrostatic imaging via conformal mapping. Inverse Prob. 18, 1659-1672 (2002).

[3] Allaire, G.: Shape Optimization by the Homogenization Method. Springer, New York, 2002. 
[4] Alt, H.W. and Caffarelli, L.A.: Existence and regularity for a minimum problem with free boundary. J. Reine Angew. Math. 325, 105-144 (1981).

[5] Beurling, A.: On free boundary problems for the Laplace equation. Sem. on Analytic Functions. Inst. for Advanced Study Princeton. 1, 248-263 (1957).

[6] Evans, L.: Partial Differential Equations. AMS, Providence 1998.

[7] Flucher, M. and Rumpf, M.: Bernoullis free-boundary problem, Qualitative theory and numerical approximation, J. Reine Angew. Math. 486, 165-204 (1997).

[8] Garabedian, P.R.: Partial Differential Equations. Wiley, New York, 1964.

[9] Haddar, H. and Kress, R.: Conformal mappings and inverse boundary value problems. Inverse Prob. 21, 935-953 (2005).

[10] Harbrecht, H. and Mitrou, G.: Stabilization of the trial method for the Bernoulli problem in case of prescribed Dirichlet data. Math. Meth. Appl. Sci. DOI: $10.1002 /$ mma.3268

[11] Harbrecht, H. and Mitrou, G.: Improved trial methods for a class of generalized Bernoulli problems. Jour. Math. Anal. Appl. 420, 177-194 (2014).

[12] Kirsch, A.: An Introduction to the Mathematical Theory of Inverse Problems, 2nd. ed. Springer, New York, 2011.

[13] Kress, R.: Integral Equations, 3rd. ed. Springer, New York, 2014.

[14] Lewy, H.. A note on harmonic functions and a hydrodynamic problem. Proc. Amer. Math. Soc. 3, 111-113 (1952).

[15] Tepper, D.E.: Free boundary problem. SIAM J. Math. Anal. 5, 841-846 (1974).

[16] Tepper, D.E.: On a free boundary problem, the starlike case. SIAM J. Math. Anal. 6, 503-505 (1975).

[17] Trefftz, E.: Über die Kontraktion kreisförmiger Flüssigkeitsstrahlen. Z. Math. Phys. 64, 34-61 (1916).

[18] Wegmann, R.: Trefftz' integral equation method for free boundary problems of potential theory. In: Numerical Treatment of Free Boundary Value Problems (J. Albrecht ed.), Birkhäuser, Stuttgart, 335-349 (1981). 